\title{
Qualidades Psicométricas da Versão Portuguesa da SOCRATES 8D numa Amostra de Heroinodependentes em Tratamento
}

\author{
Psychometric Properties of a Portuguese Version of the SOCRATES 8D: A Study \\ with a Sample of Heroin Addicts in Treatment
}

\author{
Luís Janeiro $^{1}$, Luís Faísca² e María José Lopez Miguel ${ }^{3}$
}

\begin{abstract}
Resumo
No presente estudo, adaptámos a Stage of Change Readiness and Treatment Eagerness Scale (SOCRATES, versão $8 \mathrm{D}$ ) à língua portuguesa com o objetivo de analisar a sua estrutura fatorial e as suas propriedades psicométricas. A escala foi aplicada a uma amostra constituída por 100 adultos, maioritariamente heroinodependentes, em tratamento ambulatório ou com internamento. A Análise de Componentes Principais das respostas permitiu identificar três dimensões - Reconhecimento do Problema, Ação e Ambivalência -, evidenciando uma estrutura fatorial semelhante à originalmente proposta por Miller e Tonigan (1996). Os índices de consistência interna e estabilidade temporal para estas medidas foram adequados. $\mathrm{O}$ estudo da validade externa revelou que os resultados das subescalas diferenciavam contextos de tratamento e se associavam à avaliação dos heroinodependentes acerca das expectativas de tratamento e da equipa técnica. Assim, a versão portuguesa da SOCRATES 8D reúne as características psicométricas adequadas para avaliação da motivação para o tratamento em toxicodependentes.
\end{abstract}

Palavras-chave: motivação para o tratamento, tratamento da toxicodependência, heroinodependentes, SOCRATES, análise psicométrica

\begin{abstract}
In the present study, we adapted the Stage of Change Readiness and Treatment Eagerness Scale (SOCRATES, version 8D) to the European Portuguese and we examined its factor structure and psychometric properties. The scale was applied to a sample of 100 adults, mostly heroin addicts, in outpatient or inpatient treatment. A Principal Component Analysis applied to the data revealed three dimensions - Problem Recognition, Taking Steps and Ambivalence -, corresponding almost exactly to the factor structure originally proposed by Miller and Tonigan (1996). The reliability indices for SOCRATES three subscales (internal consistency and temporal stability) were adequate. External validity study showed that the subscales differentiated treatment contexts and were associated to the participants' evaluation regarding the treatment expectations and the technical team. Thus, the Portuguese version of SOCRATES $8 \mathrm{D}$ seems to possess the proper psychometric properties to evaluate drug addicts' treatment motivation.
\end{abstract}

Keywords: treatment motivation, drug addiction treatment, heroin addicts, SOCRATES, psychometric analysis

Este trabalho foi parcialmente suportado pela Fundação da Ciência e Tecnologia (Portugal) através do financiamento ao CBMR (referência: UID/BIM/04773/2013 CBMR 1334).

\footnotetext{
${ }^{1}$ Doutorado em Psicologia Clínica e da Saúde pela Universidade de Sevilha. Professor Auxiliar do Departamento de Psicologia e Ciências da Educação da Universidade do Algarve. Universidade do Algarve - Campus de Gambelas Faro, 8005 139, Portugal. Tel.: 96 2445624. E-mail: ljaneiro@ualg.pt

${ }^{2}$ Doutorado em Psicologia pela Universidade do Algarve. Professor Auxiliar do Departamento de Psicologia e Ciências da Educação e investigador do CBMR - Centro de Investigação Biomédica da Universidade do Algarve. Universidade do Algarve - Campus de Gambelas Faro, 8005 139, Portugal. Tel.: 96 8882194. E-mail: lfaisca@ualg.pt

${ }^{3}$ Doutorada em Psicologia Clínica e da Saúde pela Universidade de Sevilha. Professora Auxiliar do Departamento de Personalidade, Avaliação e Tratamentos Psicológicos da Universidade de Sevilha. Rua Camilo José Cela, S/N, Sevilha, 41018, Espanha. Tel.: 690940334. E-mail: mjmiguel@us.es 


\section{Introdução}

A motivação para o tratamento tem sido considerada um fator fundamental no processo de mudança das pessoas com perturbações devidas ao uso de substâncias (Collins, Malone, \& Larimer, 2012). O modelo transteórico, proposto em 1982 por Prochaska e DiClemente, constituiu um importante contributo para o estudo da motivação no tratamento destas perturbações, tendo mesmo surgido uma abordagem terapêutica apoiada especificamente neste modelo para incrementar a motivação para a mudança - a entrevista motivacional (Miller \& Rollnick, 1998). O reconhecimento crescente da importância em considerar os níveis de motivação na terapêutica das toxicodependências levou à proliferação de instrumentos para medir a motivação para a mudança de acordo com o modelo transteórico (e.g., University of Rhode Island Change Assessment, de McConnaughy, Prochaska e Velicer, 1983; Readiness to Change Questionnaire, de Rollnick, Heather, Gold e Hall, 1992; ou a Stages of Change Readiness and Treatment Eagerness Scale, SOCRATES, de Miller \& Tonigan, 1996). No entanto, nenhum desses instrumentos foi adaptado ao Português Europeu. Por este motivo, o trabalho que aqui apresentamos tem como principal objetivo a adaptação de uma escala de avaliação da motivação para a mudança (com base no modelo transteórico), a SOCRATES.

De acordo com o modelo transteórico, existe motivação para o tratamento quando a pessoa reconhece que tem um problema, não está ambivalente em relação à mudança, já realizou algumas alterações no comportamento problema e tenciona mudar no prazo de 30 dias; considera-se ainda que existe motivação para o tratamento quando a pessoa iniciou e mantém uma ação orientada para a mudança ou para a manutenção da mudança (DiClemente \& Prochaska, 1998). Desta forma, Prochaska e DiClemente (1982) consideraram que a motivação para a mudança incluía componentes como o reconhecimento do problema, a ambivalência e uma dimensão comportamental relacionada com a mobilização para a ação. A conjugação destas três componentes permite definir a motivação para a mudança do indivíduo ou, segundo a designação do modelo transteórico, o seu estádio de mudança. Indivíduos no estádio pré-contemplativo caracterizam-se pela resistência às pressões externas para mudar, por possuírem uma atitude defensiva em relação à dependência e não considerarem seriamente a hipótese de mudar nos próximos seis meses. Os contemplativos consideram seriamente a hipótese de mudar nos próximos seis meses, pois reconhecem possuir um problema, mas não se encontram preparados para mudar, dada a presença de conflito entre os prós e os contras do seu comportamento. Os que consideram seriamente a possibilidade de mudar nos próximos 30 dias e que efetuaram alguma tentativa de mudança no último ano, tendo já iniciado pequenas mudanças em relação ao seu comportamento, encontram-se no estádio designado de preparação. No estádio de ação encontram-se aqueles que desenvolveram esforços para atingir um objetivo concreto - a abstinência num período mínimo de seis meses. A manutenção refere-se ao período de tempo durante o qual é necessário atuar de modo eficaz para lidar com as situações problema e reduzir a possibilidade de recaída (Prochaska, DiClemente, \& Norcross, 1992; Prochaska \& Prochaska, 1993; Prochaska \& Norcross, 2003). Este percurso ao longo dos estádios de mudança do modelo transteórico tem sido interpretado como correspondendo à evolução da motivação para o tratamento (Belding, Iguchi, Lamb, Lakin, \& Terry, 1995; Drieschner, Lammer, \& Staak, 2004).

Aos estádios de mudança associa-se o constructo balanço decisional (Rollnick, Mason, $\&$ Butler, 2005), ao qual DiClemente (2003) atribuiu uma função de marcador da mudança e de progressão nos estádios. O balanço decisional avaliação dos prós e contras em relação ao comportamento problema e à mudança do mesmo - é importante sobretudo no estádio contemplativo e de preparação nos quais se esboça a intenção de mudança, pois os prós em relação a esta aumentam progressivamente quando a pessoa considera a hipótese de mudar, e os contras diminuem, alcançando-se um ponto de cruzamento no estádio de preparação para a 
mudança (DiClemente, 2003; Prochaska et al.,1994).

\section{Motivação para a mudança, resultados e processo terapêutico}

A forma como a motivação para a mudança foi conceptualizada pelo modelo transteórico levou a que ocorressem modificações significativas na prática clínica. Tradicionalmente, os psicólogos assumiam que as pessoas com problemas devido ao uso de substâncias se encontravam em fase de ação desde que solicitassem ajuda. Por esse motivo, as intervenções que lhes eram propostas pressupunham a sua disponibilidade para mudar. Prochaska e Prochaska (1993) defenderam que o terapeuta, ao considerar que todos aqueles que iniciam o tratamento se encontram no estádio de ação, corre o risco de propor tarefas terapêuticas inadequadas e até contraproducentes. Assim, no início do tratamento dever-se-ia avaliar sempre o estádio de mudança dos clientes (Joe, Simpson \& Broome, 1998). Essa avaliação inicial da motivação é fundamental para as decisões que determinam o processo terapêutico (e.g. Connors, Donovan \& DiClemente, 2001; Derisley \& Reynolds, 2000), nomeadamente ao nível da planificação das intervenções terapêuticas específicas para efetuar a passagem ao estádio seguinte (DiClemente, Schlundt, \& Gemmell, 2004; DiClemente \& Prochaska, 1998) e em termos do estabelecimento da relação terapêutica (Prochaska \& Norcross, 2003). Por exemplo, a um cliente que está no estádio contemplativo é inadequado sugerir tarefas de tratamento que impliquem um compromisso de abstinência e assumir uma relação demasiado diretiva; para este cliente seriam adequadas tarefas que o ajudem a consciencializar os prós e contras da mudança e, numa relação de diálogo socrático, a criar uma discrepância entre os objetivos autodefinidos e os obstáculos à sua concretização que resultam do seu comportamento de dependência (Miller \& Rollnick, 1999).

Paralelamente, os estudos empíricos revelaram o importante contributo da motivação para a obtenção de resultados terapêuticos (Prochaska et al., 1992; Prochaska \& Prochaska, 1993). Prochaska et al. (1994) encontraram uma relação direta entre os resultados obtidos no tratamento e o estádio de mudança em que a pessoa se encontrava à entrada do mesmo, tendo verificado que após 18 meses estavam abstinentes somente $6 \%$ dos participantes que, ao início, se encontravam no estádio pré-contemplativo, enquanto a percentagem de abstinência para aqueles que iniciaram o tratamento no estádio contemplativo ou de preparação subia, respetivamente, para $15 \%$ e $24 \%$. Derisley e Reynolds (2000) verificaram também que as pessoas que se encontravam no estádio de contemplação no início da terapia terminavam prematuramente o tratamento. Tais resultados vão ao encontro do defendido por Prochaska e Norcross (2003) ao afirmarem que o estádio de mudança em que a pessoa se encontra no início do tratamento determina a amplitude das mudanças observadas. Outros estudos revelaram a associação entre variáveis subjacentes aos estádios de mudança (nomeadamente, o reconhecimento do problema e a mobilização para a ação) e os consumos de álcool. Por exemplo, uma maior severidade do consumo de álcool no início do tratamento associa-se a níveis mais elevados de reconhecimento do problema (Bertholet, Dukes, et al., 2009); a uma maior mobilização para a ação correspondem melhores resultados em termos de consumos de álcool em avaliações de follow-up (Bertholet, Cheng, Palfai, Samet, \& Saitz, 2009; Maisto et al., 2011).

Diversos estudos empíricos têm também demonstrado que o efeito da motivação na obtenção de resultados pode ser mediado por variáveis do processo terapêutico. DiClemente, Bellino e Neavins (1999) propuseram que a estreita relação entre o estádio de mudança ao início do tratamento e os resultados terapêuticos obtidos pode ser mediada pela formação precoce de uma aliança terapêutica por parte dos utentes mais motivados. Isto é, a motivação no início do tratamento pode potenciar a aliança terapêutica e estes dois fatores, em conjunto, determinam melhores resultados. Joe, Simpson, Greener e Rowan-Szal (1999) advogaram, também, que a motivação no início do tratamento influencia de modo indireto e positivamente a relação terapêutica. Gossop, Stewart e Marsden (2003) verificaram este efeito de mediação ao 
constatarem que a perceção da relação com a equipa técnica mediava a influência da motivação na diminuição dos consumos de heroína em clientes integrados em programa de metadona. Em suma, a motivação para a mudança parece determinar os resultados obtidos no tratamento, tanto de forma direta como indiretamente através da relação terapêutica (Joe et al., 1998).

\section{Motivação, severidade da dependência e contexto de tratamento}

Os toxicodependentes em tratamento com internamento avaliam o seu percurso de dependência como mais grave e problemático do que os toxicodependentes em tratamento ambulatório. $\mathrm{Na}$ realidade, os toxicodependentes em contexto residencial ou em internamento apresentam maior severidade no padrão de consumo de substâncias, mais problemas relativamente ao percurso criminal e ao acesso aos serviços de saúde (Yih-Ing, Anglin, \& Fletcher, 1998), mais problemas psicológicos (D’Anna \& Pérez, 2004) e familiares (Schmidt, 2001) e mais problemas recentes de consumo de álcool e drogas (Harrison \& Asche, 1999). Este tipo de percurso problemático determina muitas vezes o grau de reconhecimento da dependência e da motivação para o tratamento (Shen, McLellan, \& Merril, 2000). Assim, o contexto de tratamento, ao refletir a severidade da dependência, poderá definir grupos de participantes com características motivacionais distintas. De facto, Gossop, Stewart e Marsden (2007) verificaram que os toxicodependentes em tratamento residencial apresentavam maiores consumos de heroína, mais ansiedade e depressão, sendo também os que mais reconheciam os seus problemas de consumo.

\section{Estudos sobre a SOCRATES 8D}

Dado o poder preditivo da motivação ao nível dos resultados terapêuticos e a sua importância para a planificação do tratamento ao cliente, Drieschner et al. (2004) sublinharam a necessidade de criar instrumentos de medida da motivação para o tratamento. A "Stages of Change Readiness and Treatment Eagerness Scale” (SOCRATES; Miller \& Tonigan, 1996) foi considerada uma medida de referência para avaliação da motivação para o tratamento (Kelly
\& Greene, 2014) como, aliás, demonstram os diversos estudos de adaptação desta escala realizados em países culturalmente muito distintos (Chun, Cho, \& Shin, 2010; Evren, Dalbudak \& Çakmak, 2008; Demmel, Beck, Richter, \& Reker, 2004; Figlie, Dunn, \& Laranjeira, 2005; Kobayashi et al., 2010; Yeh, 2009; Zullino et al., 2007).

A primeira versão da escala SOCRATES foi concebida em 1987 por William Miller (Miller \& Tonigan, 1996) com o objetivo de avaliar em qual dos cinco estádios de mudança se encontravam os indivíduos com problemas de álcool em tratamento. Depois de diversos estudos, Miller e Tonigan (1996) concluem que a SOCRATES não identifica estádios de mudança mas avalia a motivação para a mudança através de três dimensões independentes e subjacentes a esses estádios. A análise fatorial das respostas à SOCRATES permitiu identificar três fatores: $a c ̧ a \tilde{o} o$ (8 itens), reconhecimento do problema (7 itens) e ambivalência (4 itens), explicando 27\%, $11 \%$ e $7 \%$, respetivamente, da variância total das respostas. Estes fatores foram replicados numa situação de teste-reteste com dois dias de diferença e as medidas de fidelidade a eles associados foram adequadas, variando entre .85 (fator reconhecimento do problema) e .96 (fator ação).

Outros autores (Figlie et al., 2005; Maisto, Conigliaro, McNeil, Kraemer, O'Connor, \& Kelley, 1999; Maisto, Chung, Cornelius, \& Martin, 2003) estudaram a estrutura fatorial da SOCRATES e sugeriram que uma estrutura bifatorial, reunindo num mesmo fator itens relativos ao reconhecimento do problema e à ambivalência, seria mais adequada do que a solução em três fatores.

Diversos motivos têm sido apontados para explicar as diferenças encontradas relativamente à estrutura fatorial da SOCRATES. Enquanto alguns autores justificam a instabilidade do fator ambivalência ao nível conceptual, considerando a possibilidade de ambivalência e reconhecimento do problema constituírem duas faces do mesmo fenómeno (Figlie et al., 2005), essa mesma instabilidade pode ser atribuída a especificidades da escala, nomeadamente a reduzida consistência interna deste fator (Maisto et al., 2003) ou a 
inadequação do conteúdo dos itens que o avaliam (Lopes, Delgado, Gamito, \& Trigo, 2010). Por outro lado, problemas relacionados com a tradução dos itens para línguas muito distintas (como, por exemplo, o Alemão, o Turco, o Coreano; Demmel et al., 2004; Evren et al., 2008; Chun et al., 2010), bem como a diversidade dos procedimentos estatísticos utilizados nos estudos fatoriais da SOCRATES (abordagem fatorial exploratória ou confirmatória, diferentes critérios para extrair e identificar fatores, interpretações baseadas em soluções ortogonais e nãoortogonais) podem ter contribuído para as diferentes soluções fatoriais propostas. Finalmente, o facto das populações estudadas provirem de diferentes contextos de tratamento e apresentarem problemas com características heterogéneas poderá influenciar o modo como o fator ambivalência se manifesta nas respostas dos participantes (Burrow-Sanchez \& Lundberg, 2007).

Pelo acima referido, o estudo psicométrico da SOCRATES continua a suscitar interesse devido à diversidade de resultados encontrados relativamente à sua estrutura fatorial.

\section{Objetivo}

Apesar da ampla disseminação da SOCRATES, apenas se conhece um estudo de adaptação à língua portuguesa (do Brasil) que investiga a estrutura fatorial da escala (Figlie et al., 2005). No referido estudo, no processo de tradução os autores alteraram a formulação e a modalidade de resposta de três itens (itens 13, 14 e 19), decisão que poderá ter influenciado os resultados da análise fatorial, a qual foi favorável a uma solução bifatorial. Por outro lado, não existe uma versão da SOCRATES adaptada ao Português Europeu. Embora a versão de Figlie et al. (2005), após alguns ajustamentos culturais ao vocabulário utilizado na escala, tenha sido já aplicada a participantes portugueses (Lopes, et al., 2010), consideramos ser necessário proceder a uma adaptação formal da escala SOCRATES (Miller \& Tonigan, 1996) à população portuguesa. Assim, no presente estudo, os procedimentos de adaptação envolveram:
1. Tradução e adaptação da versão original da escala SOCRATES 8D (Miller \& Tonigan, 1996) para o Português Europeu;

2. Estudo da estrutura fatorial da escala;

3. Análise da consistência interna e da estabilidade temporal dos fatores;

4. Estudo da validade externa da escala:

a. Análise da sua sensibilidade para detetar diferenças nas expectativas positivas em relação ao tratamento e na representação da equipa técnica em função do estádio de mudança;

b. Análise da sua sensibilidade para avaliar diferenças na motivação para o tratamento em função do contexto de tratamento (tratamento em ambulatório e tratamento em regime de internamento ou sócio-terapêutico).

\section{Método}

\section{Participantes}

No estudo que contribuiu para adaptação da SOCRATES 8D para a população portuguesa participaram 100 toxicodependentes em tratamento, 85 do sexo masculino e 15 do sexo feminino, com idades compreendidas entre os $19 \mathrm{e}$ os 52 anos $(M=33 ; D P=7,0)$. A maioria $(92 \%$ da amostra) possuía escolaridade ao nível do Ensino Básico ( $1^{\circ}$ ciclo: $26 \%$; $2^{\circ}$ ciclo: $45 \%$; $3^{\circ}$ ciclo: $21 \%)$.

Os participantes foram recrutados por conveniência em três tipos de tratamentos: em ambulatório, em comunidade terapêutica e em regime sócio-terapêutico, representando respetivamente $52 \%, 33 \%$ e $15 \%$ da amostra.

Relativamente ao tipo de acompanhamento farmacológico dos participantes, a amostra distribui-se pelos programas de substituição, com o programa de metadona a representar $50 \%$ e o de buprenorfina $13 \%$ - com uma dose média de 38,6 mg $(D P=23,9)$ e de $4,6 \mathrm{mg} \quad(D P=2,9)$, respetivamente; o programa livre de drogas representa $34 \%$ da amostra e programa com antagonista apenas 3\%. A heroína foi referida por $86 \%$ dos participantes como a droga que mais problemas lhes trouxe ao longo da vida, seguida pela cocaína (7\%) e pelo "rebolau" (4\%). No que respeita ao padrão de consumos nos últimos 30 dias, $33 \%$ dos participantes referiu consumos de haxixe com uma frequência média de 3 a 5 vezes 
por semana, enquanto $12 \%$ referiram consumos de álcool, $12 \%$ consumos de heroína e 12\% consumos de cocaína, com consumos médios de 3 a 5 vezes por semana para o álcool e heroína e de 1 a 2 vezes por semana para a cocaína.

\section{Instrumentos}

\section{Entrevista estruturada}

Foi concebida uma entrevista estruturada com o objetivo de recolher informação para a caracterização sociodemográfica dos entrevistados, avaliar o padrão de consumos ao longo da vida e nos últimos 30 dias, e conhecer o tratamento atual e os efetuados anteriormente.

\section{SOCRATES 8D}

A SOCRATES 8D destina-se ao estudo da motivação para o tratamento na população consumidora de drogas e resultou da adaptação do conteúdo dos itens da SOCRATES 8A, originalmente dirigida à população consumidora de álcool. A SOCRATES 8D inclui 19 itens avaliados numa escala de resposta com cinco níveis (entre "1 - Não! Discordo fortemente" e "5 - Sim! Concordo fortemente") e que descrevem o modo como o respondente se pode sentir face ao seu uso de drogas; os valores das respostas dadas aos diferentes itens são somados para obter as pontuações de cada subescala.

A tradução da SOCRATES 8D obedeceu a duas metodologias distintas: a técnica cega paralela e a retroversão, tal como descritas por Behling e Law (2000). Deste modo, dois tradutores fizeram a tradução da escala de forma independente, reuniram-se e chegaram a uma versão de consenso. Esta versão foi entregue a um terceiro tradutor que a retroverteu para Inglês. As três versões (original, tradução e retroversão) foram comparadas e reavaliadas, tendo os tradutores e investigador chegado à versão portuguesa final.

Avaliação de expectativas em relação ao tratamento e representação da equipa técnica

Foi construída uma escala com o objetivo de avaliar as expectativas positivas em relação ao tratamento (cinco itens, por exemplo, "com este tratamento vou alcançar os meus objetivos") e a representação da equipa técnica (dois itens, por exemplo, "acredito na qualidade e competência desta equipa técnica"). Os sujeitos respondiam aos itens através de uma escala de cinco níveis (de 1 - discordo fortemente a 5 - concordo fortemente), correspondendo a pontuação das duas subescalas à média dos respetivos itens. Uma análise da fidelidade revelou que tanto a medida de expectativas positivas como a medida de representação da equipa técnica possuíam uma consistência interna adequada (alpha de Cronbach: .829 e .877 , respetivamente) e uma estabilidade temporal moderada (correlação testereteste: .651 e .681 , respetivamente).

\section{Procedimentos}

Utilizou-se como técnica de seleção dos participantes a amostragem por conveniência, ou seja, foram convidados a participar, sem qualquer critério prévio de seleção, todos os utentes que estavam disponíveis em espaço de consulta. Em simultâneo, foi-lhes proporcionada toda a informação necessária para tomarem uma decisão consciente sobre a sua participação no estudo.

Os utentes em tratamento ambulatório eram encaminhados pelos terapeutas para um gabinete, onde os instrumentos foram aplicados individualmente. Na comunidade terapêutica e no regime sócio-terapêutico, a entrevista era realizada individualmente mas as escalas eram aplicadas em grupo. A entrevista individual demorou em média entre 10 a 15 minutos, sendo conduzida pelo investigador de modo diretivo. A aplicação das escalas durou entre 15 e 20 minutos e, sempre que necessário, as pessoas com mais dificuldades em responder foram ajudadas.

$\mathrm{O}$ intervalo de tempo entre teste e reteste foi uma semana, tendo-se aplicado a SOCRATES 8D a metade dos participantes $(n=51)$.

\section{Procedimento estatístico}

Os dados foram previamente verificados tendo-se constatado a presença de dez não respostas. Dada a reduzida presença de respostas omissas $(10 / 1900=0,53 \%)$, estas foram estimadas pelo método EM - Expectation Maximization (IBM - SPSS, versão 23).

Recorremos à Análise de Componentes Principais (PCA) por ter sido o procedimento de 
análise fatorial exploratória mais utilizado nos estudos da estrutura interna da SOCRATES. Seguindo também os procedimentos utilizados em estudos anteriores, a interpretação dos fatores foi realizada após rotação Varimax. Desta forma, pretendemos garantir uma maior comparabilidade dos resultados obtidos com a adaptação portuguesa da SOCRATES 8D.

\section{Resultados}

\section{Estrutura fatorial}

O primeiro passo no estudo da estrutura fatorial da SOCRATES 8D consistiu em avaliar a adequação dos dados a este tipo de análise. $\mathrm{O}$ índice $\mathrm{KMO}$ foi satisfatório $(\mathrm{KMO}=.727)$ e o teste de Bartlett foi significativo $\left(X^{2}=698,1, g l=171\right.$, $p<.001)$, resultados que indicam que a matriz de correlações é adequada para ser sujeita a análise fatorial exploratória. As respostas dos inquiridos foram então sujeitas a uma análise de componentes principais, da qual resultaram oito componentes com um valor próprio superior a 1. No entanto, a inspeção do scree plot, a análise paralela de Horn (o quarto componente aleatório apresenta um eigenvalue médio com valor 1,44 , superior ao valor do quarto componente obtido a partir da matriz real de dados, 1.22) e a interpretação dos fatores, após rotação varimax, sugere uma solução de três fatores, a que corresponde uma variância explicada de 47,8\%.

À exceção do item 5, que não se associa a nenhuma das três componentes (saturação <.3), todos os restantes apresentam saturações superiores a .4 apenas numa das componentes identificadas (Quadro 1). Pelo motivo indicado o item 5 foi excluído das análises posteriores.

A primeira componente (valor próprio $=4,79$ ) contribui em 25,2\% para a variância dos resultados e contém seis itens, todos eles associados na escala original ao fator Reconhecimento do Problema. Em relação a este fator da escala original, apenas o item 1 foi excluído da componente que aqui identificamos, surgindo agora ligado à nossa segunda componente.

$\mathrm{Na}$ segunda componente (valor próprio $=2.51$ ), a contribuir em $13.2 \%$ para a variância, existem oito itens, todos eles provenientes do fator Ação da escala original, à exceção do item 1, proveniente do fator Reconhecimento do Problema.

A terceira componente (valor próprio $=1,78$ ) explica $9.4 \%$ da variância e reúne os quatro itens do fator Ambivalência da escala original.

Uma vez que as componentes identificadas quase se sobrepõem aos da escala original, decidimos manter os nomes que lhe foram atribuídos por Miller e Tonigan (1996), isto é, Reconhecimento do Problema (componente 1), Ação (componente 2) e Ambivalência (componente 3 ).

A correlação entre as pontuações obtidas nas subescalas correspondentes às três componentes são reduzidas apesar de estatisticamente significativas (Reconhecimento do Problema e Ação: $\quad r=.27, \quad p=.007 ; \quad$ Reconhecimento do Problema e Ambivalência: $r=.28, p=.005$; Ação e Ambivalência: $r=.29, p=.004$ ), o que aponta para a relativa independência das dimensões obtidas pela versão portuguesa da SOCRATES 8D.

\section{Fidelidade}

No que diz respeito à fidelidade das medidas, utilizaram-se dois índices, o alpha de Cronbach e a correlação de Pearson para avaliação da estabilidade temporal (Quadro 2).

A componente Reconhecimento do Problema possui os valores mais elevados em qualquer um dos índices de fidelidade, revelando excelentes consistência interna (alpha>.8, tanto no teste como no reteste) e estabilidade temporal ( $r=.82$; $p<.001)$.

No que respeita à componente Ação, obtiveram-se resultados satisfatórios ao nível da consistência interna (alpha=.7) e resultados mais moderados relativamente à estabilidade teste $\mathrm{e}$ reteste $(r=.54 ; p<.001)$.

Embora a componente Ambivalência possua menor número de itens, o que poderia diminuir a sua consistência interna, os valores da fidelidade são aceitáveis no que respeita à consistência interna e à estabilidade.

Por último, não se verificaram diferenças significativas na comparação dos valores médios por componente na situação de teste e reteste, indicando estabilidade temporal dos níveis de resposta a estas subescalas. 
Quadro 1. Estrutura Fatorial da SOCRATES 8D: Saturações fatoriais e comunalidades

Itens da SOCRATES 8D

\begin{tabular}{clr} 
Escala & \multicolumn{2}{l}{ Saturação fatorial } \\
\cline { 2 - 3 } original & $h^{2}$ & RP A Amb
\end{tabular}

\section{Fator 1 - Reconhecimento do problema}

10. Eu tenho um problema sério com drogas.

15. Eu sei que tenho um problema com as drogas.

7. Eu tenho um problema com drogas.

12. O meu consumo de substâncias está a causar muito mal.

17. Eu sou um toxicodependente.

3. Se não mudar rapidamente em relação ao meu consumo de drogas os meus problemas vão tornar-se piores.

5. Em tempos já consumi demasiado, mas consegui alterar isso.

Fator 2 - Ação

14. Eu quero ajuda para evitar ter os mesmos problemas que antes tinha com o consumo de drogas.

9. Eu já mudei em relação ao consumo de drogas e procuro aprender maneiras que me ajudem a evitar recaídas.

4. Eu já comecei a fazer algumas mudanças em relação ao consumo de drogas.

1. Eu realmente quero mudar em relação ao consumo de drogas.

18. Estou a esforçar-me para mudar em relação ao consumo de drogas.

13. Atualmente estou fazendo coisas para parar ou diminuir o meu consumo de drogas.

8. Não estou só a pensar em mudar em relação às drogas, já estou de facto a fazer algo por isso.

19. Fiz algumas mudanças em relação ao consumo de drogas e quero ajuda para evitar consumir drogas do mesmo modo que consumia antes.

\section{Fator 3 - Ambivalência}

2. Às vezes pergunto a mim mesmo se sou dependente das drogas.

6. Às vezes pergunto-me se o facto de eu consumir magoa as outras pessoas.

16. Às vezes pergunto-me se o meu consumo de drogas é excessivo.

$\begin{array}{lllll}\mathrm{RP} & .86 & .08 & .03 & .75\end{array}$

$\begin{array}{lllll}\mathrm{RP} & .86 & .20 & .08 & .79\end{array}$

$\begin{array}{lllll}\mathrm{RP} & .83 & .09 & -.07 & .70\end{array}$

$\begin{array}{lllll}\mathrm{RP} & .72 & .13 & .17 & .56\end{array}$

$\begin{array}{lllll}\mathrm{RP} & .72 & -.02 & .11 & .53\end{array}$

$\begin{array}{lllll}\mathrm{RP} & .58 & .11 & .40 & .50\end{array}$

$\begin{array}{lllll}\mathrm{A} & -.29 & .04 & .25 & .14\end{array}$

A

$\begin{array}{lllll}\mathrm{A} \quad & .08 & .74 & -.08 & .55\end{array}$

A

$\begin{array}{llll}-.15 & .69 & -.08 & .50\end{array}$

A

$\begin{array}{lllll}\mathrm{A} & -.34 & .58 & .09 & .46\end{array}$

$\begin{array}{lllll}\mathrm{RP} & .19 & .56 & -.02 & .35\end{array}$

$\begin{array}{lllll}\mathrm{A} & .23 & .51 & .24 & .37\end{array}$

A

$\begin{array}{llll}.25 & .47 \quad .18 \quad .32\end{array}$

A

$\begin{array}{llll}.30 & .42 & .19 & .30\end{array}$

A

$\begin{array}{llll}.05 & .41 & .35 & .30\end{array}$

11. Às vezes pergunto a mim mesmo se tenho controlo sobre o meu consumo de drogas.

$\begin{array}{lllll}\mathrm{Amb} & -.03 & .00 & .76 & .60 \\ \mathrm{Amb} & -.08 & .15 & .72 & .55 \\ \mathrm{Amb} & .34 & .08 & .61 & .50 \\ \mathrm{Amb} & .16 & .05 & .56 & .34\end{array}$

Nota. RP - Reconhecimento do Problema; A - Ação; Amb - Ambivalência.

\section{Validade externa}

Para estudar a validade externa da SOCRATES 8D foram utilizadas as medidas de expectativas positivas em relação ao tratamento e de representação da equipa, anteriormente descritas. Os participantes foram classificados num estádio de mudança em função da pontuação obtida nas subescalas Reconhecimento do Problema e Ação da SOCRATES, formando quatro grupos: a) os pré-contemplativos (Reconhecimento do Problema e Ação abaixo da média do grupo; $\mathrm{n}=27)$; b) os contemplativos (Reconhecimento do Problema acima da média do grupo e Ação abaixo da média; n=20); os pertencentes ao estádio de ação (Reconhecimento do Problema e Ação acima da média do grupo; $\mathrm{n}=42$ ); e os que se encontravam em manutenção (Reconhecimento do Problema abaixo da média 
Quadro 2. Fidelidade da SOCRATES 8D

\begin{tabular}{lcccccc}
\hline & \multicolumn{2}{c}{ alpha de Cronbach } & \multicolumn{3}{c}{ Teste-Reteste $(\mathrm{n}=51)$} \\
\cline { 2 - 6 } & $\begin{array}{c}\text { Teste } \\
(\mathrm{n}=100)\end{array}$ & $\begin{array}{c}\text { Reteste } \\
(\mathrm{n}=51)\end{array}$ & $r$ & \multicolumn{2}{c}{ Teste $t$} \\
\cline { 5 - 7 } & .870 & .824 & $.82 * * *$ & .70 & .489 \\
\hline $\begin{array}{l}\text { Reconhecimento do } \\
\text { Problema }\end{array}$ & .691 & .714 & $.54 * * *$ & 1.28 & .208 \\
Ação & .665 & .690 & $.67 * * *$ & .79 & .432 \\
Ambivalência & & &
\end{tabular}

Nota. ${ }^{* * *} p \leq, 001$ (teste bilateral)

do grupo e Ação acima da média; n=11). Posteriormente, averiguámos se a classificação dos participantes com base nos estádios de mudança introduziu diferenças significativas nas expectativas positivas em relação ao tratamento e na representação da equipa técnica.

No que respeita às expectativas positivas, verificámos que existiam diferenças significativas entre estádios (teste de Kruskal-Wallis, $H=12.02$; $p=.007)$. Uma análise à posteriori das diferenças revelou-nos que o grupo que se encontra no estádio pré-contemplativo apresenta expectativas positivas significativamente mais baixas do que o grupo em ação (teste Mann-Whitney, $U=358.0$; $p=.010)$ e do que o grupo em manutenção $(U=59.0 ; p=.003)$. O grupo em contemplação distingue-se apenas do grupo em manutenção ( $U=55,0 ; p=.023$ ). Como se pode observar através da Figura 1(a) à medida que se progride nos estádios de mudança aumentam as expectativas positivas em relação ao tratamento.

O comportamento da variável representação da equipa técnica é semelhante ao que se verificou em relação às expectativas positivas, embora as diferenças sejam apenas marginalmente significativas $(H=6.92 ; p=.075)$. Assim, o grupo pré-contemplativo distingue-se tanto do grupo em ação $(U=396.5 ; p=.030)$ como do grupo em manutenção $(U=83.5 ; p=.035)$. Tal como nas expectativas positivas em relação ao tratamento, a representação da equipa técnica é mais elevada à medida que se progride nos estádios (Figura 1b).

A análise da validade externa das medidas fornecidas pela SOCRATES recorreu também às diferenças atribuídas aos contextos de tratamento, contrastando o tratamento em ambulatório $(n=52)$ com o tratamento em comunidade terapêutica ou sócio-terapêutico $(\mathrm{n}=48)$.
Os resultados permitiram verificar que o grupo de pessoas com um tratamento que exige um contexto terapêutico mais restritivo e contentor (comunidade terapêutica ou programa sócio-terapêutico) reconhece mais o problema e tem um número médio de tratamentos superior ao grupo a efetuar tratamento em ambulatório. Estes resultados confirmam a sensibilidade da escala para discriminar participantes em função do contexto de terapêutico.

\section{Discussão}

A análise da estrutura fatorial da versão portuguesa da SOCRATES 8D no grupo de participantes toxicodependentes em tratamento confirma a estrutura de três fatores ortogonais sugerida por Miller e Tonigan (1996). Ao comparar a solução factorial obtida no presente estudo com proposta por Miller e Tonigan (1996), encontramos valores de variância explicada muito semelhantes $(48 \%$ e $45 \%$, respetivamente). No entanto, a representatividade dos fatores altera-se, pois a componente Reconhecimento do Problema ganha no nosso estudo especial destaque (explica $25 \%$ em vez dos $11 \%$ do estudo original), a componente Ação passa a explicar $13 \%$ em vez dos $27 \%$ e o componente Ambivalência passa a explicar $9 \%$ e não $7 \%$. As diferenças verificadas na representatividade destes componentes podem dever-se ao tipo de população estudada, uma vez que a amostra portuguesa é maioritariamente constituída por consumidores de heroína e os participantes do estudo realizado por Miller e Tonigan (1996) eram alcoólicos. Tal como se verificou nos trabalhos através dos trabalhos de Maisto et al. (1999, 2003) e Burrow-Sanchez 
Quadro 3. Motivação e dados do tratamento em função do contexto de tratamento

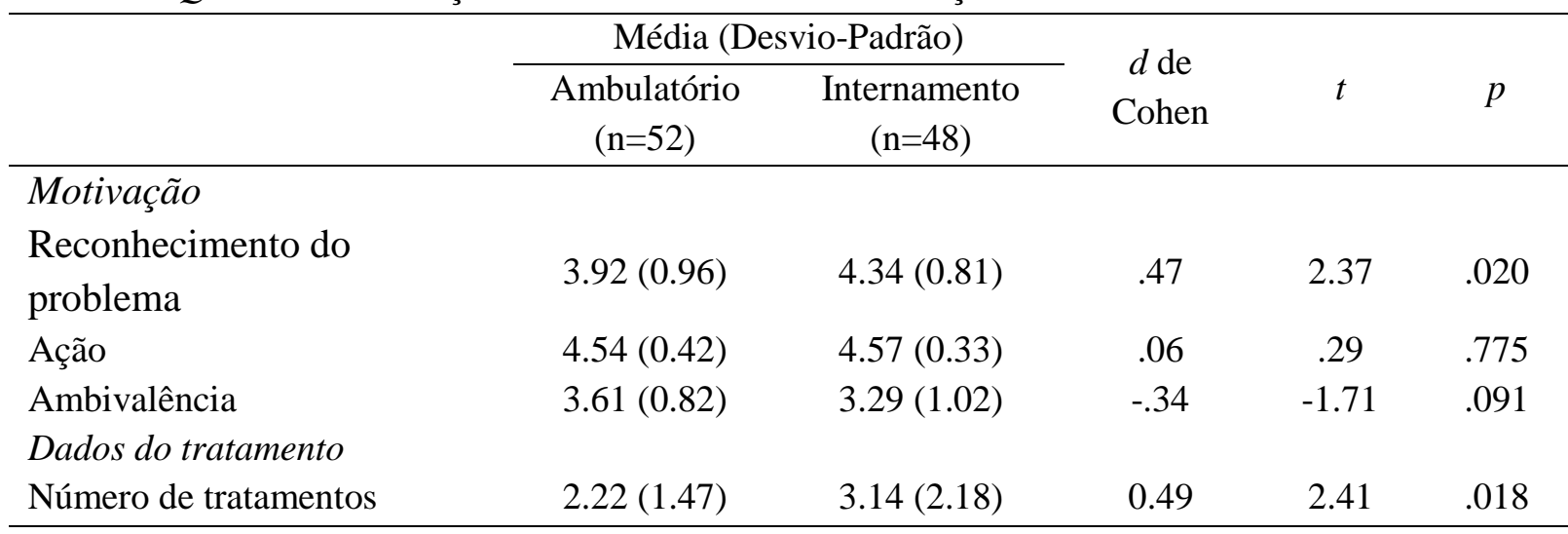

(2015), também os contextos de tratamento podem reforçar essas diferenças: encontrando-se a maioria dos participantes do nosso estudo em programa de substituição (63\%), no qual é permitida a manutenção de consumos, o componente Reconhecimento do Problema adquire maior variabilidade, variando entre os que realmente reconhecem o problema e param consumos e os que não reconhecem o problema e mantêm os consumos.

A associação dos itens às três componentes é clara (ausência de saturações duplas e saturações superiores a .4), com exceção do item 5 que não satura significativamente em nenhuma delas. A distribuição dos restantes itens pelas três componentes foi semelhante à obtida no estudo da versão original da SOCRATES (Miller \& Tonigan, 1996), excetuando o item 1 (“eu realmente quero mudar em relação ao consumo de drogas") originalmente alocado ao fator Reconhecimento do Problema mas integrando a componente Ação do estudo atual. Este item temse revelado problemático na maior parte dos estudos de adaptação da SOCRATES devido a saturações duplas ou por reduzir a consistência interna (Bertholet, Dukes et al., 2009; BurrowSanchez \& Lundberg, 2007; Burrow-Sanchez, 2015; Figlie et al., 2005; Yeh, 2009; Maisto et al., 2003)

Os resultados relativos à fidelidade da versão portuguesa da SOCRATES 8D são inferiores aos apresentados na versão original, embora permitam atestar a consistência interna e estabilidade temporal das componentes identificadas.

No que se refere à validade externa da SOCRATES, verificámos que as expectativas positivas em relação ao tratamento e a representação da equipa técnica se diferenciavam em função do estádio de mudança dos participantes, identificado a partir das componentes Reconhecimento do Problema e Ação. Observou-se que existia uma tendência de crescimento em ambas as variáveis em função da evolução nos estádios de mudança. No entanto, não existem diferenças significativas entre estádios adjacentes e, neste sentido, poder-se-á afirmar que a SOCRATES 8D não possui sensibilidade suficiente para descriminar claramente entre estádios próximos. Na medida em que as expectativas positivas em relação ao tratamento podem ser consideradas uma dimensão do balanço decisional - os prós em relação à mudança -, variável que se sabe evoluir positivamente a par da motivação para o tratamento (DiClemente, 2003), a diferenciação entre os estádios no nível das expectativas confirmam a validade externa da SOCRATES 8D. Também, a evolução positiva da representação da equipa técnica ao longo dos estádios de mudança parece confirmar a validade externa da escala. Estes resultados vão ao encontro do sugerido por Simpson et al. (1995) e Connors et al. (1997), que consideram que a relação com a equipa técnica se associa diretamente à motivação para o tratamento e aos resultados do mesmo.

A análise da validade externa baseou-se ainda na diferença entre contextos de tratamento, uma vez que toxicodependentes em tratamento com internamento apresentam uma maior severidade da dependência (Harrison et al. 1999; Yin-Ing et al., 1998) e tendem, por isso, a mostrar um maior reconhecimento do problema (Joe et al., 1998; Shen et al., 2000). 
Figura 1. (a) Expectativas positivas em relação ao tratamento; e (b) Representação da equipa técnica - média em cada estádio de mudança

(a)

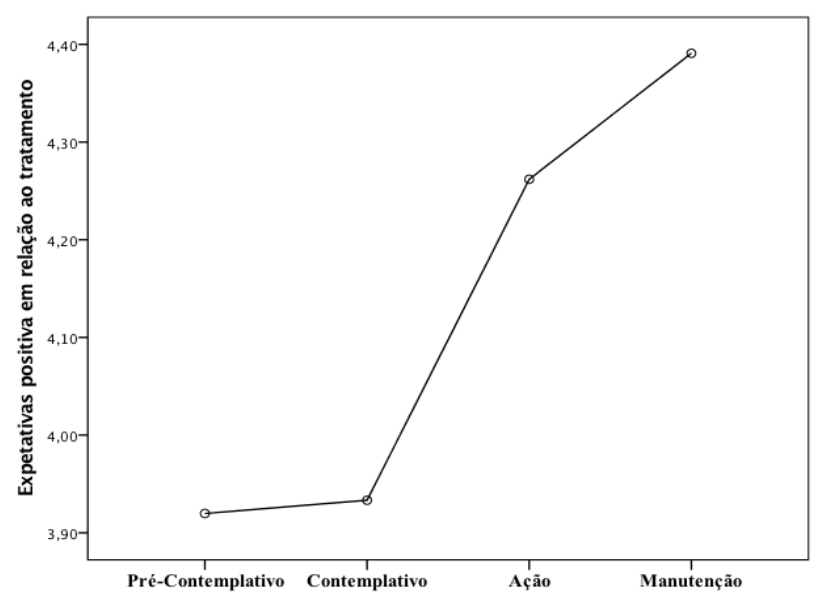

(b)

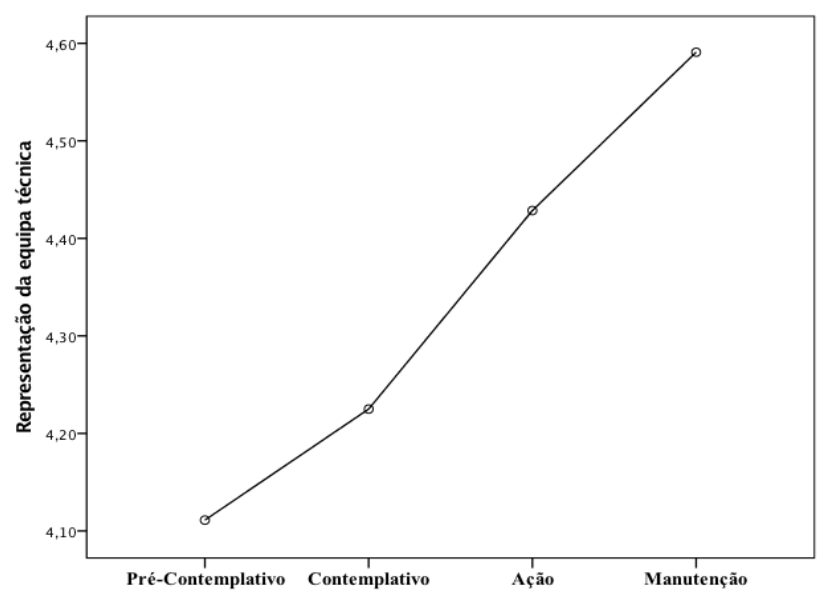

Os resultados permitiram verificar que o grupo de pessoas com um tratamento que exige um contexto terapêutico mais restritivo e contentor (comunidade terapêutica ou programa sócio-terapêutico) reconhecem mais o seu problema de dependência e tiveram maior número de tratamentos do que o grupo em ambulatório. Estes resultados confirmam a sensibilidade da escala para discriminar o nível de motivação dos participantes em função do seu contexto terapêutico.

No que se refere à interpretação das medidas proporcionadas pela SOCRATES $8 \mathrm{D}$, as três componentes identificadas deverão ser entendidas como variáveis contínuas subjacentes aos estádios de mudança, tal como sugeriram Miller e Tonigan (1996), e com uma valoração interpretativa próxima da proposta pela CASAA Research
Division (s.d.). No entanto, sendo os estádios definidos como entidades discretas, consideramos que para manter a correspondência com o modelo transteórico, a identificação do estádio de mudança de um individuo deverá basear-se na conjugação das pontuações obtidas nas subescalas da SOCRATES. Por exemplo, uma pessoa que reconhece o problema mas não atua estaria contemplativa, enquanto outra que reconhece o problema e atua se encontra em ação. Esta perspetiva interpretativa assume a relativa independência das componentes, condição verificada através dos valores reduzidos da correlação entre subescalas no nosso estudo. Assim, sugerimos a seguinte classificação nos estádios de mudança em função das pontuações no Reconhecimento do Problema e na Ação: a) no estádio pré-contemplativo encontram-se os sujeitos com baixa pontuação no Reconhecimento do Problema e Ação; b) no estádio contemplativo os sujeitos possuem uma pontuação elevada no Reconhecimento do Problema, mas baixa na Ação; c) os sujeitos que se situam no estádio ação possuem uma elevada pontuação nas subescalas Reconhecimento do Problema e Ação; d) e, no estádio manutenção situam-se os sujeitos com elevada pontuação em Ação, mas baixa pontuação no Reconhecimento do Problema. À componente Ambivalência atribuímos uma interpretação qualitativa que reflete o grau de questionamento relativo à dependência de drogas.

Contudo, nesta categorização o estádio de manutenção não está definido de acordo com o modelo transteórico, uma vez que estar em manutenção significa reconhecer o problema e manter a ação. O facto de não reconhecer e ainda assim manter uma atuação em relação ao problema poderá indicar que a pessoa não se reconhece no problema de consumo de drogas tal como explicitado em itens do tipo "eu sou toxicodependente". Na verdade, o participante pode ter parado o consumo de substâncias e manter comportamentos de prevenção da recaída como expresso no item "eu já mudei em relação ao consumo de drogas e procuro aprender maneiras que me ajudem a evitar recaídas".

Como referimos, a estrutura fatorial identificada neste estudo corresponde à estrutura trifatorial proposta em diversos estudos de adaptação da SOCRATES (Chun et al., 2010; 
Demmell et al., 2004; Evren et al., 2008; Miller \& Tonigan, 1996; Vik, Culbertson, \& Sellers, 2000; Yeh, 2009; Zullino et al., 2007), mas contraria a solução bifatorial sugerida por outros autores (Bertholet, Dukes et al., 2009; Burrow-Sanchez, 2015; Burrow-Sanchez \& Lundberg, 2007; Figlie et al., 2005; Maisto et al., 1999, 2003) que aglutinam a Ambivalência e o Reconhecimento do Problema na variável Consciência do Problema. O estudo da estrutura fatorial da versão portuguesa da SOCRATES, ao ter sido conduzido numa população adulta em tratamento, maioritariamente dependente de heroína, poderá contribuir para a caracterização dos contextos e populações onde a dimensão Ambivalência se diferencia das outras duas dimensões.

No nosso estudo estão representados diversos contextos terapêuticos, embora não tenha sido possível apresentar resultados de consumidores de drogas que não se encontrassem em tratamento. Esta limitação é recorrente nos estudos efetuados na área da toxicodependência e, em particular, na adaptação da escala SOCRATES (ver estudos de Maisto et al., 1999, 2003 e Figlie, et al., 2005). Neste sentido, e em função da distinção introduzida por DiClemente (1999) entre motivação para o tratamento e motivação para a mudança, consideramos que a SOCRATES 8D mede a motivação para o tratamento.

Apesar da escala utilizada como critério externo para o estudo da validade da SOCRATES 8D garantir as características psicométricas aceitáveis para a sua utilização, constatamos que outras medidas de validade externa como, por exemplo, os marcadores de mudança (balanço decisional e auto-eficácia), ou listas de classificação do terapeuta sobre adesão e a aliança terapêutica, seriam úteis em estudos posteriores sobre a motivação para o tratamento, e em particular no que respeita à validação de medidas de avaliação na população toxicodependente de acordo com o modelo transteórico.

Por último, apesar da dimensão da amostra respeitar o mínimo exigido para a análise de componentes principais que realizámos, uma amostra mais alargada permitiria estimar valores normativos para as três componentes e, assim, determinar de forma mais fiável os critérios de demarcação dos estádios de mudança.
Em conclusão, o estudo das características psicométricas da adaptação da SOCRATES 8D à língua portuguesa e aplicada a uma amostra de toxicodependentes em tratamento confirma a estrutura fatorial da escala original de Miller e Tonigan (1996), tendo-se obtido índices de fidelidade satisfatórios e demonstrado a sua validade externa, resultados que permitem a utilização desta escala em contexto clínico e de investigação. As componentes identificadas devem ser entendidas como variáveis contínuas subjacentes ao processo de mudança, podendo a sua interpretação ajustar-se ao modelo transteórico através da conjugação dos valores obtidos pelos sujeitos nas subescalas Reconhecimento do Problema e Ação.

\section{Referências}

D’Anna, A., \& Pérez, M. (2004). Clinical profile of drug dependents asessed with MCMI-III. Revista Iberoamericana de Diagnóstico y Evaluación - e Avaliação Psicológica, 18(2), 29-42.

Behling, O., \& Law, K. S. (2000). Translating questionnaires and other research instruments: Problems and solutions. Thousand Oaks: Sage.

Belding, M. A., Iguchi, M. Y., Lamb, R.J., Lakin, M., \& Terry, R. (1995). Stages an processes of change among polydrug users in methadone maintenance treatment. Drug and Alcohol Dependence, 39, 45-53.

Bertholet, N., Cheng, D. M., Palfai, T. P., Samet, J. H., \& Saitz, R. (2009). Does readiness to change predict subsequent alcohol consumption in medical inpatients with unhealthy alcohol use? Addictive Behaviors, 34(8), 636-640. doi:10.1016/j.addbeh.

2009.03.034

Bertholet, N., Dukes, K., Horton, N. J., Palfai, T. P., Pedley, A., \& Saitz, R. (2009). Factor structure of the SOCRATES questionnaire in hospitalized medical patients. Addictive Behaviors, 34(6-7), 568-72. doi:10.1016/j.addbeh.2009.03.013

Burrow-Sanchez, J. J. (2015). Identifying the Factor Structure of the SOCRATES in a Sample of Latino Adolescents. Psychology of 
Addictive Behaviors, 29(6), 997-1003. doi:10.1037/a0033605

Burrow-Sanchez, J. J., \& Lundberg, K. J. (2007). Readiness to change in adults waiting for publicly funded substance abuse treatment. Addictive Behaviors, 32(1), 199-204. doi:10.1016/j.addbeh.2006.03.039

CASAA Research Division. (s.d.). SOCRATES: Stages of Change Readiness and Treatment Eagerness Scale, Consultado em 3 de Maio, 2005, de http:// casaa.unm.edu/inst/SOCRATESv8.pdf.

Chun, Y.-M., Cho, S.-M., \& Shin, S.-M. (2010). Factor structure of a Korean-language version of the Stages of Change Readiness and Treatment Eagerness Scale (SOCRATES) in a clinical sample of clients with alcohol dependence. Psychology of Addictive Behaviors, 24(4), 555-562. doi:10.1037/a0021492

Collins, S. E., Malone, D. K., \& Larimer, M. E. (2012). Motivation to change and treatment attendance as predictors of alcohol-use outcomes among project-based Housing First residents. Addictive Behaviors, 37(8), 931939. doi:10.1016/j.addbeh.2012.03.029

Connors, G.J., Donovan, D.M., \& DiClemente, C.C. (2001). Substance abuse treatment and the stages of change: Selecting and planning interventions. New York: Guilford Press.

Demmel, R., Beck, B., Richter, D., \& Reker, T. (2004). Readiness to change in a clinical sample of problem drinkers: Relation to alcohol use, self-efficacy, and treatment outcome. European Addiction Research, 10(3), 133-138. doi:10.1159/000077702

Derisley, J., \& Reynolds, S. (2000). The transtheoretical stages of change as predictor of premature termination, attendance and alliance in psychotherapy. British Journal of Clinical Psychology, 39, 371-382.

DiClemente, C. C. (2003). Addiction and change: How addictions develop and addicted people recover. New York: Guilford.

DiClemente, C. C., Bellino, L. E., \& Neavins, T. M. (1999). Motivation for change and alcoholism treatment. Alcohol Research and Health, 23(2), 86-92.

DiClemente, C.C., \& Prochaska, J.O. (1998). Toward a comprehensive, transtheoretical model of change: Stages of change and addictive behaviors. In W.R. Miller \& N. Heather (Eds.), Treating addictive behaviors $\left(2^{\text {nd }}\right.$ ed., pp.3-24). Nova York: Plenum Press.

DiClemente, C.C., Schlundt, B.S., \& Gemmell, B.S. (2004). Readiness and stages of change in addiction treatment. The American Journal on Addictions, 13, 103-119.

Drieschner, K. D., Lammers, S. M., \& Staak, C. P. (2004). Treatment motivation: An attempt for clarification of ambiguous concept. Clinical Psychology Review, 23, 1115-1137.

Evren, C., Dalbudak, E., \& Çakmak, D. (2008). Deiflime Hazir Olma ve Tedavi istegi Ölçegi (SOCRATES) Türkçe Versiyonu'nun Yatarak Tedavi Gören Erkek Alkol Bagmlisi Hastalarda Faktör Yapisi, Geçerligi ve Güvenirligi. Klinik Psikofarmakoloji Bulteni, 18, 84-91.

Figlie, N. B., Dunn, J., \& Laranjeira, R. (2005). Motivation for change in alcohol dependent outpatients from Brazil. Addictive Behaviors, 30(1), 159-65. doi:10.1016/j.addbeh.2004. 01.007

Gossop, M., Stewart, D., \& Marsden, J. (2003). Treatment process components and heroin use outcome among methadone patients. Drug and Alcohol Dependence, 71, 93-102.

Gossop, M., Stewart, D., \& Marsden, J. (2007). Readiness for change and drug use outcomes after treatment. Addiction (Abingdon, England), 102(2), 301-8. doi:10.1111/j.13600443.2006.01681.x

Harrison, P. A., \& Asche, S. E. (1999). Comparison of substance abuse treatment outcomes for inpatients and outpatients. Journal of Substance Abuse Treatment, 17(3), 207-220.

Joe, G.W., Simpson, D.D., \& Broome, K.M. (1998). Effects of readiness for drug abuse treatment on client retention and assessment of process. Addiction, 93(8), 1177-1190.

Joe, G.W., Simpson, D.D., Greener, J. M., \& Rowan-Szal, G.A. (1999). Integrative modeling of client engagement and outcomes during the first 6 months of methadone treatment. Addictive Behaviors, 24(5), 649659.

Kelly, J. F., \& Greene, M. C. (2014). Beyond motivation: Initial validation of the - Vol.2 $\cdot 177-191 \cdot 2016$ 
commitment to sobriety scale. Journal of Substance Abuse Treatment, 46(2), 257-263. doi:10.1016/j.jsat.2013.06.010

Kobayashi, O., Matsumoto, T., Chiba, Y., Imamura, F., Morita, N., \& Wada, K. (2010). Factor structure and validity of the Japanese version of SOCRATES (Stages of Change Readiness and Treatment Eagerness Scale) in adolescent delinquents placed in detention home. Nihon Arukoru Yakubutsu Igakkai Zasshi = Japanese Journal of Alcohol Studies \& Drug Dependence, 45(5), 437-451.

Lopes, P., Prieto, G., Delgado, A. R., Gamito, P., \& Trigo, H. (2010). Rasch-modeling the Portuguese SOCRATES in a clinical sample. Psychology of Addictive Behaviors: Journal of the Society of Psychologists in Addictive Behaviors, 24(2), 355-359. doi: $10.1037 / \mathrm{a} 0019080$

Maisto, S.A., Chung, T.A., Cornelius, J.R., \& Martin, C.S. (2003). Factor structure of the SOCRATES in a clinical sample of adolescents. Psychology of Addictive Behaviors, 17(2), 98-107.

Maisto, S. A., Conigliaro, J., McNeil, M., Kraemer, K., O’Connor, M., \& Kelley, M.E. (1999). Factor structure of SOCRATES in a sample of primary care patients. Addictive Behaviors, 24(6), 879-892.

Maisto, S. A., Krenek, M., Chung, T., Martin, C. S., Clark, D., \& Cornelius, J. (2011). A comparison of the concurrent and predictive validity of three measures of readiness to change alcohol use in a clinical sample of adolescents. Psychological Assessment, 23(4), 983-994. doi:10.1037/a0024136

McConnaughy, E. A., Prochaska, J. O., \& Velicer, W. F. (1983). Stages of change in psychotherapy: Measurement and sample profiles. Psycho- therapy: Theory, Research, and Practice, 20, 368-375.

Miller, W. R., \& Rollnick, S. (1999). La entrevista motivacional: Preparar para el cambio de conductas adictivas. Barcelona: Paidós.

Miller, W.R., \& Tonigan, J.S. (1996). Assessing Drinkers' Motivation for Change: The Stages of Change Readiness and Treatment Eagerness Scale (SOCRATES). Psychology of Addictive Behaviors, 10(2), 81-89.
Prochaska, J. O., \& DiClemente, C. C. (1982). Transtheoretical therapy: Toward a more integrative model of change. Psychotherapy: Theory, Research and Practice, 19(3), 276288.

Prochaska, J. O., DiClemente, C.C., \& Norcross, J.C. (1992). In search of how people change: Applications to addictive behaviors. American Psychologist, 47(9), 1102-1114.

Prochaska, J.O., \& Norcross, J.C. (2003). Systems of psychotherapy: A transtheoretical analysis. Pacific Grove: Thomson.

Prochaska, J. O., Norcross, J. C., \& DiClemente, C. C. (1994). Changing for good. New York: HarperCollins.

Prochaska, J.O., \& Prochaska, J.M. (1993). Modelo transteórico de cambio para conductas adictivas. In M.C. Brugué \& M. Gossop (Eds.), Tratamentos psicológicos en drogodependências: Recaída e prevención de recaidas (pp.85-136). Barcelona: Citran.

Rollnick, S., Heather, N., Gold, R., \& Hall, W. (1992). Development of a short Readiness to Change Questionnaire for use in brief, opportunistic interventions among excessive drinkers. British Journal of Addiction, 87, 743-754.

Rollnick, S., Mason, P., \& Butler, C., (1999). Health Behavior Change: A Guide for Practitioners. New York: Churchill Livingstone.

Schmidt, V. (2001). Recursos para el afrontamiento de eventos vitales estresantes en Familias de Drogodependientes Coping resources of life stressors in drug dependent's families. Revista Iberoamericana de Diagnóstico y Evaluación - e Avaliação Psicológica, 11(1), 75-92.

Shen, Q., McLellan, A.T., \& Merril, J.C. (2000). Client's perceived need for treatment and its impact on outcome. Substance Abuse, 21(3), 179-92.

Vik, P. W., Culbertson, K. A., \& Sellers, K. (2000). Readiness to change drinking among heavy-drinking college students. Journal of Studies on Alcohol, 61(5), 674-680.

Yeh, M. Y. (2009). A psychometric evaluation of the Chinese version of the stage of changereadiness and treatment-eagerness scale. 
Journal of Clinical Nursing, 18(17), 24942502. doi:10.1111/j.1365-2702.2008.02592.x

Yih-Ing, H., Anglin, M.D., \& Fletcher, B. (1998). Comparative treatment effectiveness: Effects of program modality and client drug dependence history on drug use reduction. Journal of Substance Abuse Treatment, 15(6), 513-523.

Zullino, D. F., Krenz, S., Frésard, E., Montagrin, Y., Kolly, S., Chatton, A., Manghi, R. A. \& Broers, B. (2007). Psychometric properties of a French-language version of the stage of change readiness and treatment eagerness scale (SOCRATES). Addiction Research \& Theory, 15(2), 153-160. doi:10.1080/16066350601179514 